\title{
The effects of different pilot-drilling methods on the mechanical stability of a mini-implant system at placement and removal: a preliminary study
}

\author{
Il-Sik Cho, MSD, ${ }^{a}$ HyeRan Choo, MSD, ${ }^{\text {b }}$ Seong-Kyun Kim, DDS, MSD, PhD, ${ }^{c}$ \\ Yun-Seob Shin, MSD, ${ }^{\mathrm{d}}$ Duck-Su Kim, DDS, MSD, PhD, ${ }^{\mathrm{e}}$ Seong-Hun Kim, DDS, MSD, PhD, ${ }^{\mathrm{g}}$ \\ Kyu-Rhim Chung, DDS, MSD, PhD, ${ }^{\mathrm{f}}$ John C. Huang, DDS, MSD, PhD ${ }^{\mathrm{h}}$
}

\begin{abstract}
Objective: To investigate the effects of different pilot-drilling methods on the biomechanical stability of self-tapping mini-implant systems at the time of placement in and removal from artificial bone blocks. Methods: Two types of artificial bone blocks (2-mm and 4-mm, 102-pounds per cubic foot [102-PCF] polyurethane foam layered over 100-mm, 40-PCF polyurethane foam) were custom-fabricated. Eight mini-implants were placed using the conventional motor-driven pilot-drilling method and another 8 mini-implants were placed using a novel manual pilot-drilling method (using a manual drill) within each of the 2-mm and 4-mm layered blocks. The maximum torque values at insertion and removal of the mini-implants were measured, and the total energy was calculated. The data were statistically analyzed using linear regression analysis. Results: The maximum insertion torque was similar regardless of block thickness or pilot-drilling method. Regardless of the pilot-drilling method, the maximum removal torque for the 4-mm block was statistically higher than that for the 2-mm block. For a given block, the total energy at both insertion and removal of the mini-implant for the manual pilot-drilling method were statistically higher than those for the motor-driven pilot-drilling method. Further, the total energies at removal for the 2-mm block was higher than that for the 4-mm block, but the energies at insertion were not influenced by the type of bone blocks. Conclusions: During the insertion and removal of mini-implants in artificial bone blocks, the effect of the manual pilot-drilling method on energy usage was similar to that of the conventional, motor-driven pilot-drilling method. (Korean J Orthod 2011;41(5):354-360)
\end{abstract}

Key words: Anchorage, Implant design, Surface treatment, Orthodontic mini-implant

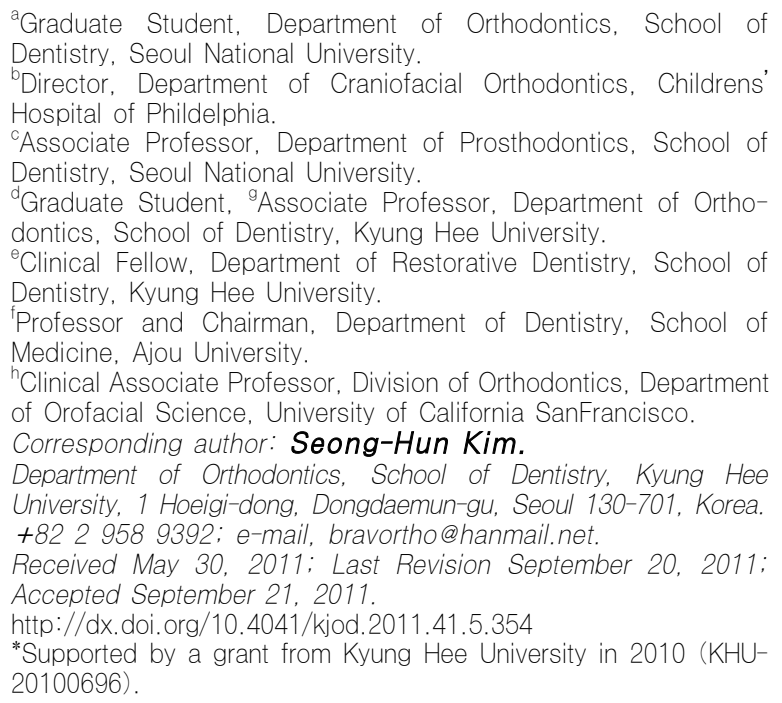


pitches and a screw apex for self-tapping only and often has a two-piece design with a surface treatment that promotes osseointegration during its use in orthodontic therapy. ${ }^{6-8}$

The C-implant system is a pilot-drilling self-tapping screw system. Conventionally, this system requires a motor-driven pilot-drill prior to the insertion of the self-tapping mini-screw body. ${ }^{7,9}$ The C-implant is a two-piece system by design (i.e. the head and the screw body are separate, giving the flexibility of selection of the head component after placing the screw body in the optimal recipient site). The C-implant system was originally developed as a common orthodontic tool to be placed and utilized by orthodontists. However, the requirement of a motor-driven pilot-drilling procedure prior to screw placement has always been a significant technical and psychological burden to the orthodontists, despite the well-documented long-term stability of the system (utilization of osseointegrationpromoting surface treatment) and the inherent procedural safety during the self-tapping screw placement (Fig 1). ${ }^{7,10,11}$ Consequently, orthodontists often have had to refer patients to a periodontist or oral surgeon for C-implant mini-screw placement, with the result that the use of the $\mathrm{C}$-implant system has become unnecessarily cumbersome and less practical for patients and orthodontists.

The trepidation for using the motor-driven pilot-drilling procedure further increased after the Centre for
Disease Control and Prevention (CDC) defined any dental protocols requiring a motor-driven pilot-drilling as an oral surgical procedure. ${ }^{12}$ Therefore, according to CDC standards, the current C-implant placement system is considered an oral surgical procedure, which is outside the realm of most orthodontic insurance liability coverage. On the other hand, the CDC does not categorize a self-drilling mini-screw placement (without a tissue punch incision) as an oral surgical procedure. Instead, this procedure is considered a common dental procedure equivalent to a local anesthetic injection, making the procedure more amenable to most orthodontists.

To abide by the CDC regulation that defines the scope of common dental procedures for orthodontists and to eliminate orthodontists' fear for performing an "oral surgery" procedure in an orthodontic clinical setting, the authors recently developed a method using a manual drill prior to the insertion of the conventional C-implant mini-screw body (Fig 2). Preliminary clinical observations indicate that the novel manual, pilot-drilling method will be successful in replacing the conventional motor-driven pilot-drilling procedure without compromising the biomechanical stability of the Cimplant system at time of placement and removal. Therefore, the current in vitro study utilized the C-implant system as an exemplary pilot-drilling self-tapping orthodontic mini-screw to investigate differences in the biomechanical properties of self-tapping orthodontic
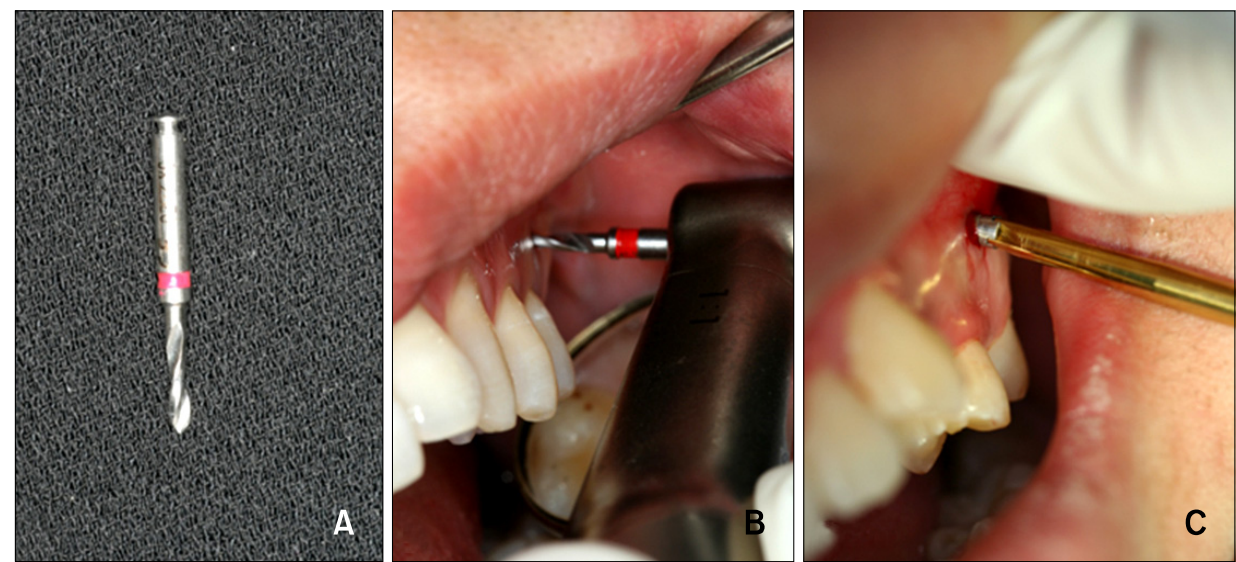

Fig 1. Motor-driven pilot-drilling method. A, A $1.5 \mathrm{~mm}$ diameter guide drill; B, pilot drilling using a guide drill; C, C-implant placement using a hand driver. 

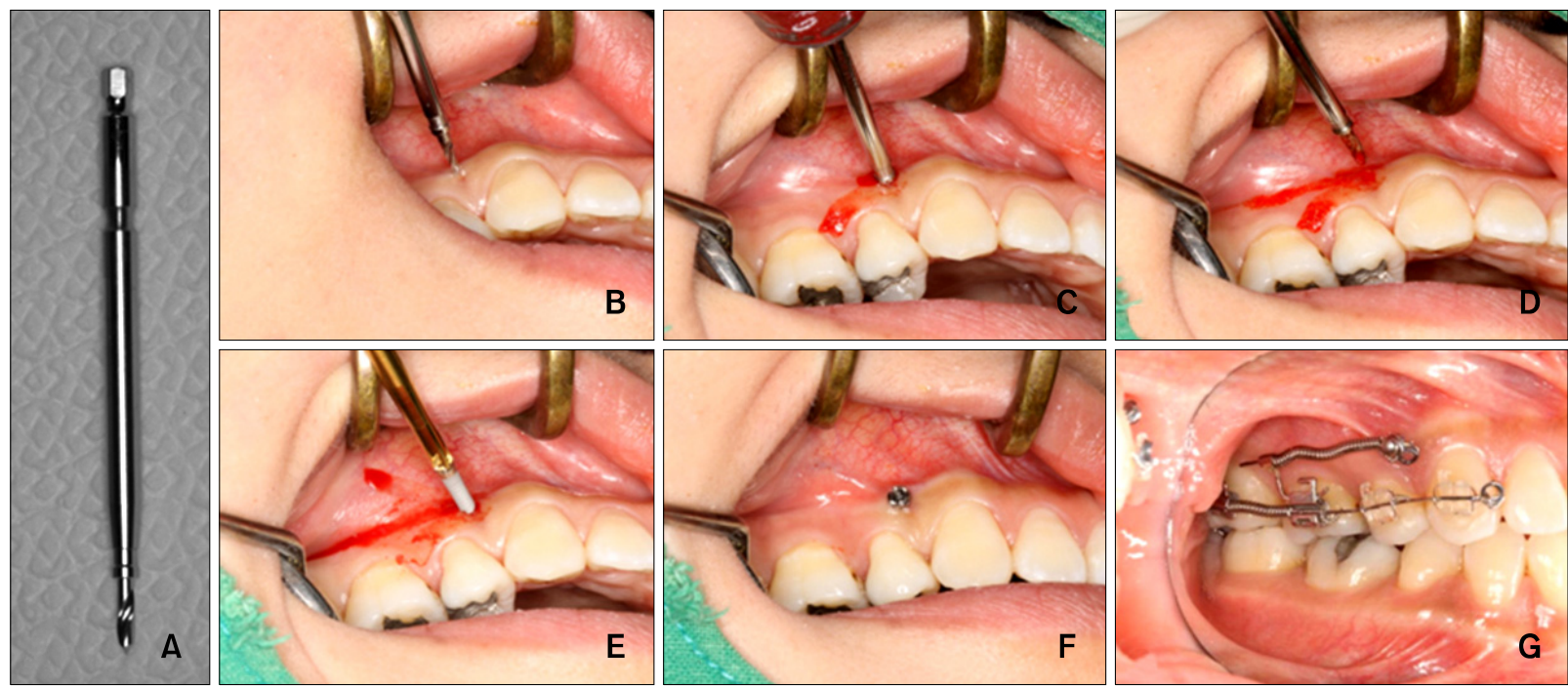

Fig 2. Manual pilot-drilling method using a hand drill. A, A $1.5 \mathrm{~mm}$ diameter hand drill; $\mathbf{B}$ and $\mathbf{C}$, hand-drilling on the planned positions of a mini-implant placement; $\mathbf{D}$, removal of a hand drill with a counter clockwise rotation; $\mathbf{E}$ and F, C-implant placement on the pilot hole; $\mathbf{G}$, after adaption of the head part.

mini-screws at placement and removal when using different pilot-drilling procedures at placement. The influence of varying densities at the recipient sites was also compared. We hypothesized that the maximum insertion and removal torque values, angular momentum, and total energy of C-implant placement and removal with different pilot-drilling methods are not different.

\section{MATERIAL AND METHODS}

Two types of artificial bone blocks with different densities were custom-fabricated using polyurethane foams (Sawbones ${ }^{\circledR}$, Pacific Research Laboratories Inc., Vashon, WA, USA) (Fig 3). Both types of blocks had a base of 100-mm, 40-pounds per cubic foot (PCF) polyurethane foam, but the first type of block was covered with a 2-mm sheet of 102-PCF polyurethane foam and named as 2-mm block. The cover of the second type of block was a 4-mm sheet of 102-PCF polyurethane foam, and this block was named as 4-mm block.

Eight C-implants were placed in the 2-mm block by the conventional motor-driven pilot-drilling method (Group A). Another $8 \mathrm{C}$-implants were placed in the 2-mm block by the novel manual pilot-drilling method using a hand-drill (Group B). The manual drills were $5 \mathrm{~mm}$
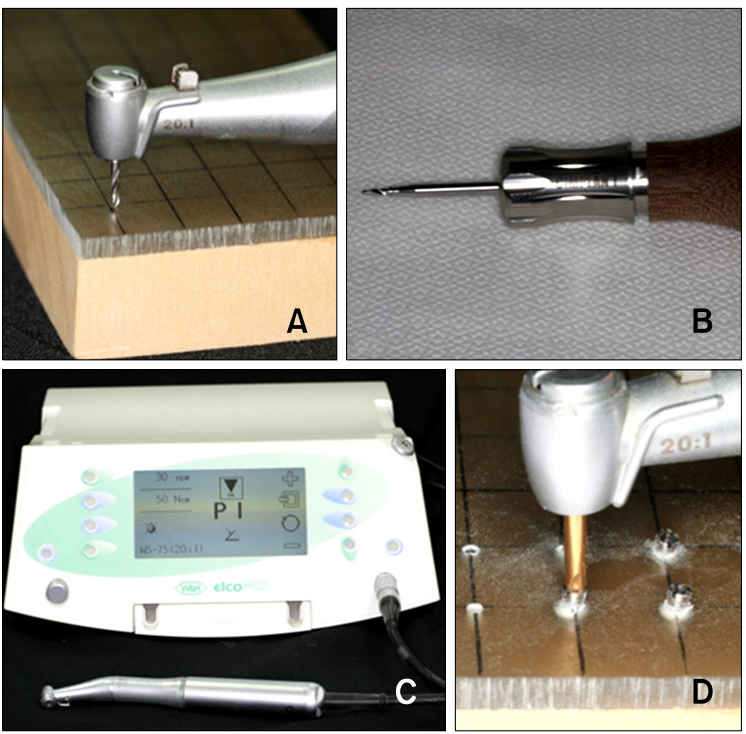

Fig 3. A, Motor-driven pilot-drilling procedure on a artificial bone block; B, a hand drill for manual pilot drilling; $\mathbf{C}$ and $\mathbf{D}$, a surgical engine implant system for the insertion of mini-implants and measurement of insertion and removal torques.

long and $1.6 \mathrm{~mm}$ in diameter. Further, $8 \mathrm{C}$-implants were placed in the 4-mm block by the conventional pilot-drilling method (Group C), and $8 \mathrm{C}$-implants were placed in the 4-mm block by the novel pilot-drilling method (Group D). The C-implants used in all the 4 


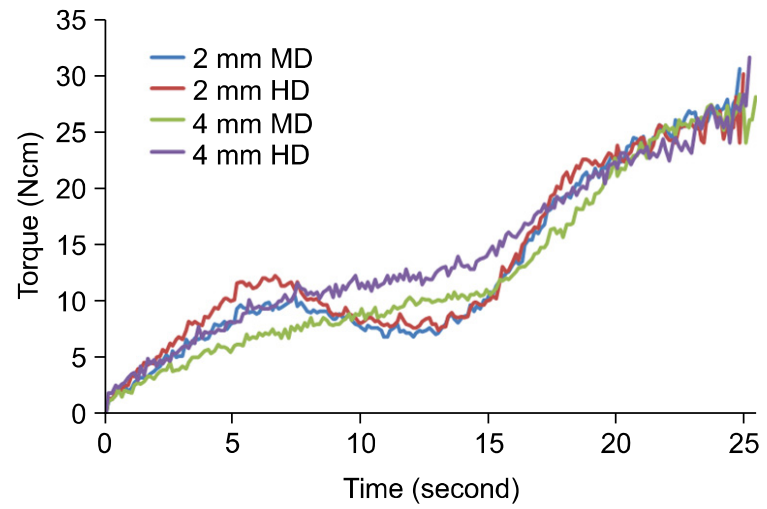

Fig 4. Graph showing the insertion torques with respect to the elapsed time. MD, Motor-driven pilot drilling; HD, manual pilot-drilling using a hand drill.

groups were $6.5 \mathrm{~mm}$ long and $1.8 \mathrm{~mm}$ in diameter. All C-implants were placed perpendicularly at the block surface. The depth of the pilot-drill was $5 \mathrm{~mm}$. Slight hand pressure was imposed on the pilot drill to make indentations of $2 \mathrm{~mm}$ and $4 \mathrm{~mm}$ on the 2-mm block and 4-mm block, respectively. Perpendicular hand pressure is not necessary up to the 5-mm pilot-drilling depth. Immediately following the designated pilot-drilling procedure in each group, a C-implant was engaged in a surgical engine (Elcomed SA200C; W\&H, Burmoos, Austria), and the torque values at the time of C-implant placement and removal were measured in continuous mode. The rotational speed of the engine was fixed at 30 rotations per minute (rpm) ( 0.5 rotations per second), and the maximum torque value possible to measure was set at $50 \mathrm{~N} \cdot \mathrm{cm}$ both at insertion and removal. The C-implants were placed in the artificial bone block to its maximum length $(6.5 \mathrm{~mm})$ and then removed completely in a continuous mode.

In the study, we were interested in the maximum torque values, total energy, and angular momentum at insertion and removal of the $\mathrm{C}$-implants by the 2 pilotdrilling methods. Briefly, the maximum torque value was defined as the highest numeric value measured during the time of C-implant mini-screw placement or removal (in $\mathrm{N} \cdot \mathrm{cm}$ ). The total energy (J) was calculated from the measured torque values, using a Javabased customized computer program, as the total sum of energy used to place or remove the C-implant to or from its maximum length. ${ }^{11,13}$ The values of total en-

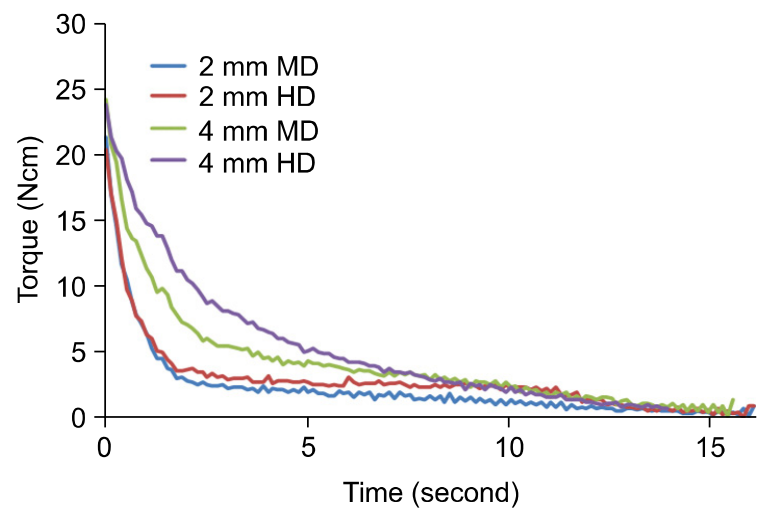

Fig 5. Graph showing the removal torques with respect to the elapsed time. MD, Motor-driven pilot drilling; HD, manual pilot-drilling using a hand drill.

ergy and angular momentum were positively correlated in a mathematical equation, resulting in a similar pattern of results.

\section{Statistical analysis}

Linear regression analysis was used to compare the effects of the 2 pilot-drilling methods in each artificial bone block. Regardless of inclusion of the 2 interaction factors (type of artificial bone block and pilot-drilling method) in linear analyses, no statistical difference was found for all 4 parameters (maximum insertion torque, total insertion energy, maximum removal torque, and total removal energy). Therefore, a linear model with a higher degree of freedom, without the inclusion of interaction factors, was selected as the final statistical model for the current study.

\section{RESULTS}

The numeric values of each parameter are shown in Table 1. The value of maximum insertion torque did not show any correlation either with the type of artificial bone block or with the pilot-drilling method (Fig 4). The value of angular momentum of insertion did not show statistically significant correlation with the artificial block quality. However, for the same type of artificial block, the total insertion energy when the manual pilot-drilling method was used was statistically higher than that when the motor-driven pilot drilling 
Table 1. Maximum torque $(\mathrm{Ncm})$ and total energy $(\mathrm{J})$ employed during insertion and removal for SLA surface-treated orthodontic mini-implants in relation to different thicknesses of sawbone and type of pilot drilling

\begin{tabular}{|c|c|c|c|c|}
\hline & \multirow{2}{*}{$\begin{array}{l}\text { Thickness } \\
\text { (mm) }\end{array}$} & \multicolumn{2}{|c|}{ Type of pilot-drilling } & \multirow[b]{2}{*}{ Significance } \\
\hline & & $\begin{array}{l}\text { Motor driven } \\
\text { pilot-drill }(\mathrm{MD})\end{array}$ & $\begin{array}{l}\text { Hand-drill } \\
\text { (HD) }\end{array}$ & \\
\hline \multirow{2}{*}{$\begin{array}{l}\text { Maximum insertion } \\
\text { torque }(\mathrm{Ncm})\end{array}$} & 2 & $29.63 \pm 1.27$ & $28.63 \pm 0.79$ & \multirow[t]{2}{*}{ NS } \\
\hline & 4 & $29.75 \pm 1.34$ & $29.50 \pm 1.81$ & \\
\hline \multirow{2}{*}{$\begin{array}{l}\text { Total insertion } \\
\text { energy }(\mathrm{J})\end{array}$} & 2 & $9.32 \pm 0.77$ & $9.64 \pm 0.60$ & \multirow[t]{2}{*}{$\mathrm{MD}<\mathrm{HD}^{*}(p<0.05)$} \\
\hline & 4 & $9.44 \pm 0.58$ & $10.25 \pm 0.73$ & \\
\hline \multirow{2}{*}{$\begin{array}{l}\text { Maximum removal } \\
\text { torque }(\mathrm{Ncm})\end{array}$} & 2 & $21.31 \pm 2.91$ & $20.38 \pm 1.13$ & \multirow[t]{2}{*}{ Thickness $2 \mathrm{~mm}<4 \mathrm{~mm}^{\ddagger}(p<0.001)$} \\
\hline & 4 & $24.19 \pm 2.37$ & $23.75 \pm 1.98$ & \\
\hline \multirow{2}{*}{$\begin{array}{l}\text { Total removal } \\
\text { energy }(\mathrm{J})\end{array}$} & 2 & $1.15 \pm 0.13$ & $1.46 \pm 0.31$ & \multirow{2}{*}{$\begin{array}{l}\text { Thickness } 2 \mathrm{~mm}<4 \mathrm{~mm}^{\ddagger}(p<0.001) \\
\mathrm{MD}<\mathrm{HD}^{\dagger}(p<0.01)\end{array}$} \\
\hline & 4 & $2.07 \pm 0.48$ & $2.45 \pm 0.36$ & \\
\hline
\end{tabular}

SLA, Sand blasted with large grit, and acid etched; NS, not significance. ${ }^{*} p<0.05 ;{ }^{\dagger} p<0.01 ;{ }^{\dagger} p<0.001$.

method was used. As long as the same pilot-drilling method was used, the type of artificial bone block did not influence the total insertion energy.

Regardless of the pilot-drilling methods at insertion, all parameters tested at the removal of the C-implant (maximum removal torque and total removal energy) from the 4-mm block were statistically higher than those tested at removal of C-implant from the 2-mm block (Fig 5). In addition, the total removal energy was statistically higherwhen the manual pilot-drilling method was used than when the motor-driven pilot drilling method was used on the same type of block.

\section{DISCUSSION}

The C-implant system is well-known for its utilization of an osseointegration-promoting surface treatment on the mini-screw body., ${ }^{7,14}$ Despite the concerns of potentially high removal torque values of partially osseointegrated mini-screws (i.e. difficulty in removing the mini-screw when it is no longer needed for the orthodontic therapy), the advantages of partial osseointegration of orthodontic mini-screws have been wellstudied and documented. ${ }^{15,16}$ In brief, the failure rate of mini-screw systems that purely rely on mechanical retention during orthodontic therapy is significantly higher than that for systems utilizing both osseointegration and mechanical retention. Furthermore, partial osseoin- tegrated mini-screws have higher capability to withstand high levels of orthodontic force and rotational moments. ${ }^{7,11}$ One of the common findings in studies of orthodontic mini-screw systems that were stably used for an extended period of orthodontic application is that the surface of those mini-screw systems always showed some degree of osseointegration. ${ }^{8,15,16}$ To date, no studies have reported any clinical situations where partially osseointegrated orthodontic mini-screws could not be removed by conventional unscrewing methods. However, the required motor-driven pilot drill to place the C-implant system has been a significant obstacle to an otherwise easy application of C-implant systems by orthodontists. $^{12}$ Therefore, the current study was initiated to investigate the feasibility of a manual pilot drilling technique using a hand-drill in an effort to replace the need for motor-driven pilot-drilling.

We found that the maximum insertion torque did not differ statistically for either the type of artificial bone block or the type of pilot-drilling method. The maximum removal torque for removal from the 4-mm block was statistically higher than that for removal from the 2-mm block, but this parameter did not significantly differ for the type of pilot-drilling method used at insertion. For the same type of block, the total energy at both insertion and removal of the C-implant system when the manual pilot-drilling method was used were statistically higher than when the motor- 
driven pilot-drilling method was used. The total removal energy was higher for the 4-mm block than for the 2-mm block, but the total insertion energy was not influenced by the type of block.

The increased values of total energy when the manual pilot-drilling method was used can be explained by the fact that manual pilot drilling eliminates the negative effect of the operational tremor of high rpm on pilot-hole formation. The more closely adaptable pilothole made by manual pilot drilling, therefore, must have generated higher friction between the artificial bone block and the C-implant mini-screw body, increasing the total energy at both insertion and removal.

Interestingly, the different pilot-drilling methods had no influence on the maximum torque values at insertion or at removal. However, the patterns of continuous insertion torque values of the 2-mm block and 4-mm block show very distinct differences regardless of the pilot-drilling methods, as seen in Fig 4. The insertion torque value curve for the 2-mm block showed significant depression before reaching a plateau, while the curve for the 4-mm block showed a continuous increase until maximum torque values reached a plateau. The continuous torque value curves at removal showed a similar pattern regardless of the pilot-drilling method or type of artificial bone block. This may indicate that maximum insertion and removal torque values are more closely related to the architecture of the self-tapping screw than to the pilot-drilling method itself. These results are consistent with those of a recent study by Lim et al. ${ }^{17}$ that reported similar torque value curves of a cylinder-type self-tapping screw. It will be interesting to further investigate the factors that influence the maximum torque values and to determine their clinical importance on long-term effects of the C-implant system.

The quality of artificial bone block represented by the different thickness of high-density polyurethane styrofoam seems to be an important factor in estimating the resistance of the mini-screw at removal. ${ }^{18-20}$ Since the removal torque values of the current in vitro study were measured immediately following the insertion and did not take into account the biological effect of osseointegration of the self-tapping mini-screw, the maximum removal torque, total removal energy, and the angular momentum of removal estimated by an in vitro study will certainly be different from those estimated by an in vivo study. In addition, clinical variability factors such as difference of precession motion, rpm, the operative hand-gripping mode, and the pressure level during the insertion of mini-implants could be easily controlled in the current in vitro study. It should be noted that these factors can significantly vary depending on the clinical environment (visibility and bone quality) and may contribute to different outcomes depending on the setting of the in vitro study.

On the basis of the results of this study and our clinical experience, we think that for these 2 pilot-drilling methods, the biomechanical stability of mini-implants will be different primarily in the maxillary and mandibular posterior interradicular and palatal areas. Therefore, an in vivo experiment is being conducted in our laboratory to measure the same experimental parameters that were measured in the current in vitro study. A clinical trial with a sufficient sample size will also be needed to evaluate whether this manual pilotdrilling method can replace the conventional motordriven pilot-drilling method in the clinical application of orthodontic mini-implants.

\section{CONCLUSION}

The results of this in vitro study indicate that placing a C-implant system using a manual pilot-drilling method can result in biomechanical properties similar to those obtained using the conventional motor-driven pilot-drilling method; this manual method can potentially achieve even better stability as shown by the increased values of total removal energy in the artificial bone blocks. The current study warrants the need for further in vivo clinical investigations to assess the possibility of replacing the conventional motor-driven method used for placing orthodontic mini-implants with a manual method.

\section{REFERENCES}

1. Lee SY, Cha JY, Yoon TM, Park YC. The effect of loading time on the stability of mini-implant. Korean J Orthod 2008; 38:149-58.

2. Cha JY, Yoon TM, Hwang CJ. Insertion and removal torques 
according to orthodontic mini-screw design. Korean J Orthod 2008;38:5-12.

3. Yun S, Lim S. Effect of cutting flute length and shape on insertion and removal torque of orthodontic mini-implants. Korean J Orthod 2009;39:95-104.

4. Su YY, Wilmes B, Hönscheid R, Drescher D. Comparison of self-tapping and self-drilling orthodontic mini-implants: an animal study of insertion torque and displacement under lateral loading. Int J Oral Maxillofac Implants 2009;24:404-11.

5. Baumgaertel S. Predrilling of the implant site: is it necessary for orthodontic mini-implants? Am J Orthod Dentofacial Orthop 2010;137:825-9.

6. Jeon MS, Kang YG, Mo SS, Lee KH, Kook YA, Kim SH. Effects of surface treatment on the osseointegration potential of orthodontic mini-implant. Korean J Orthod 2008;38:328-36.

7. Kim SH, Cho JH, Chung KR, Kook YA, Nelson G. Removal torque values of surface-treated mini-implants after loading. Am J Orthod Dentofacial Orthop 2008;134:36-43.

8. Lee SJ, Ahn SJ, Lee JW, Kim SH, Kim TW. Survival analysis of orthodontic mini-implants. Am J Orthod Dentofacial Orthop 2010;137:194-9.

9. Park SH, Kim SH, Ryu JH, Kang YG, Chung KR, Kook YA. Bone-implant contact and mobility of surface-treated orthodontic micro-implants in dogs. Korean J Orthod 2008;38:416-26.

10. Mo SS, Kim SH, Kook YA, Jeong DM, Chung KR, Nelson G. Resistance to immediate orthodontic loading of surfacetreated mini-implants. Angle Orthod 2010;80:123-9.

11. Kim SH, Lee SJ, Cho IS, Kim SK, Kim TW. Rotational resistance of surface-treated mini-implants. Angle Orthod 2009;79: 899-907.
12. Cleveland JL, Kohn W. CDC weighs in on TADs. Am J Orthod Dentofacial Orthop 2009;136:622-3.

13. Park KJ. Relationship between implant stability quotient (ISQ values and implant insertion variables: a clinical study (thesis). Seoul: Seoul National University, 2007.

14. Kim SH, Kang SM, Choi YS, Kook YA, Chung KR, Huang JC. Cone-beam computed tomography evaluation of mini-implants after placement: is root proximity a major risk factor for failure? Am J Orthod Dentofacial Orthop 2010;138:264-76.

15. Serra G, Morais LS, Elias CN, Meyers MA, Andrade L, Müller CA, et al. Sequential bone healing of immediately loaded mini-implants: histomorphometric and fluorescence analysis. Am J Orthod Dentofacial Orthop 2010;137:80-90.

16. Cha JY, Takano-Yamamoto T, Hwang CJ. The effect of miniscrew taper morphology on insertion and removal torque in dogs. Int J Oral Maxillofac Implants 2010;25:777-83.

17. Lim SA, Cha JY, Hwang CJ. Insertion torque of orthodontic miniscrews according to changes in shape, diameter and length. Angle Orthod 2008;78:234-40.

18. Brinley CL, Behrents R, Kim KB, Condoor S, Kyung HM, Buschang PH. Pitch and longitudinal fluting effects on the primary stability of miniscrew implants. Angle Orthod 2009;79: 1156-61.

19. Chen Y, Kyung HM, Gao L, Yu WJ, Bae EJ, Kim SM. Mechanical properties of self-drilling orthodontic micro-implants with different diameters. Angle Orthod 2010;80:821-7.

20. Kim JW, Ahn SJ, Chang YI. Histomorphometric and mechanical analyses of the drill-free screw as orthodontic anchorage. Am J Orthod Dentofacial Orthop 2005;128:190-4. 